\title{
Construction and evaluation of an ultrahigh-vacuum-compatible sputter deposition source
}

\author{
Peter Lackner, ${ }^{1}$ Joong-II Jake Choi, ${ }^{1,}$ a) Ulrike Diebold, ${ }^{1}$ and Michael Schmid ${ }^{1}$ \\ Institute of Applied Physics, TU Wien, Vienna, Austria
}

(Dated: 7 March 2018)

\begin{abstract}
A sputter deposition source for use in ultrahigh vacuum (UHV) is described and some properties of the source are analyzed. The operating principle is based on the design developed by Mayr et al. [Rev. Sci. Instrum. 84, 094103 (2013)], where electrons emitted from a filament ionize argon gas, and the $\mathrm{Ar}^{+}$ions are accelerated to the target. In contrast to the original design, two grids are used to direct a large fraction of the $\mathrm{Ar}^{+}$ions to the target, and the source has a housing cooled by liquid nitrogen to reduce contaminations. The source has been used for deposition of zirconium, a material that is difficult to evaporate in standard UHV evaporators. At an Ar pressure of $9 \times 10^{-6}$ mbar in the UHV chamber and moderate emission current, a highly reproducible deposition rate of $\approx 1$ monolayer in $250 \mathrm{~s}$ was achieved at the substrate (at a distance of $\approx 50 \mathrm{~mm}$ from the target). Higher deposition rates are easily possible. X-ray photoelectron spectroscopy shows a high purity of the deposited films. Depending on the grid voltages, the substrate gets mildly sputtered by $\mathrm{Ar}^{+}$ions; in addition, the substrate is also reached by electrons from the negatively biased sputter target.
\end{abstract}

\author{
PACS numbers: \\ 81.15.Cd Deposition by sputtering \\ 61.80.Jh Ion radiation effects \\ 81.10.Pq Crystal growth in vacuum \\ 81.10.St Crystal growth in controlled gaseous atmospheres \\ Keywords: sputter deposition, thin-film growth, ultra-high vacuum
}

\section{INTRODUCTION}

Growth of (ultra-)thin films for surface-science experiments in an ultrahigh-vacuum (UHV) environment is usually performed by evaporation, using resistive heating for low to medium temperatures and electron-beam evaporation for materials that require high temperatures for evaporation. For materials that reach a sufficiently high vapor pressure below or at the melting point, electronbeam evaporation from a rod is the method of choice, having the advantage that the material is not in contact with anything that could be a source of contaminations. Other materials require temperatures well above the melting point for sufficient vapor pressures. When the end of a rod gets heated to such a high temperature, a droplet would form and detach from the rod. Thus, such materials (e.g., $\mathrm{Al}, \mathrm{Au}, \mathrm{Sn}, \mathrm{Pb}$ ) are usually evaporated from crucibles. This requires that the material must not react with the crucible at high temperatures. The crucibles of large electron-beam evaporators (kilowatt range) can be cooled while the melt is at higher temperatures, but this is not the case for small evaporators, where the electrons impinge on the crucible, not the melt. A particularly notorious material for evaporation is zirconium, which has a vapor pressure of only $\approx 4 \times 10^{-5} \mathrm{mbar}$ at the melting point $\left(\approx 1850^{\circ} \mathrm{C}\right)$, corresponding to evaporation of about two monolayers (ML) per second; the deposition rate at

\footnotetext{
a) Present address: Center for Nanomaterials and Chemical Reactions, Institute for Basic Science (IBS) and Graduate School of EEWS, Korea Advanced Institute of Science and Technology (KAIST), Daejeon 305701 , South Korea.
}

the substrate is typically lower by four orders of magnitude. Thus, evaporation from rods is very slow. $\mathrm{Zr}$ forms eutectic alloys with the refractory metals $\mathrm{Mo}, \mathrm{Ru}, \mathrm{Ta}, \mathrm{W}$, Re, and Ir, which precludes the use of metal crucibles. $\mathrm{Zr}$ also reacts with graphite crucibles. Fortunately, these problems are not relevant for sputter deposition.

In many cases, a further advantage of sputter deposition, compared to evaporation, is better layer-by-layer growth of the films. One reason for improved growth is the higher kinetic energy of the sputtered atoms (few $\mathrm{eV}$ compared to sub-eV for evaporated atoms) and also the presence of other energetic particles during sputter deposition. ${ }^{1}$ Compared to evaporation, additional advantages of sputter deposition are the possibility to grow compounds with the film composition being close to that of the sputter target, and better stability and reproducibility of the deposition rate compared to most UHV evaporators.

Conventional magnetron sputter sources using a gas discharge typically operate at pressures between $10^{-3}$ and $10^{-2}$ mbar and deposition rates of nanometers per second, which is hard to reconcile with UHV surfacescience experiments. A way out is using a standard UHVtype ion source for sputtering and collecting the sputtered material at the substrate ${ }^{1,4,5}$. This technique is sometimes referred to as ion-beam sputter deposition (IBSD) and requires a special setup of the vacuum chamber. To circumvent the rather low deposition rates achieved with standard (electron-impact ionization) UHV ion sources, high-current ion sources such as duoplasmatron or Kaufman sources can be used. ${ }^{4,5} \mathrm{~A}$ much simpler approach is a dedicated UHV-compatible sputter deposition source, as recently designed by Mayr et al. for deposition of $\mathrm{Zr}^{2,3}$. This source was also found to be useful for oxides, e.g. 


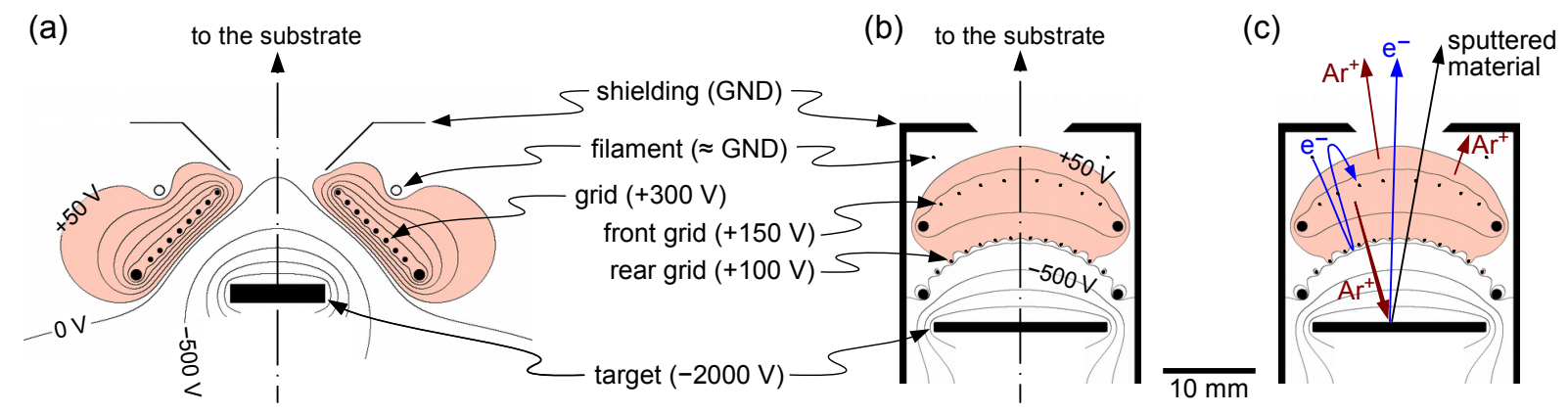

FIG. 1. Cross sections and equipotential lines (ignoring space-charge effects) of (a) the original sputter source design by Mayr et $a^{2,3}$ and (b) the design presented in this work. Schematic particle trajectories are shown in (c). The light-red shading shows the region where the electron energy exceeds $50 \mathrm{eV}$; this is roughly the volume where efficient ionization of Ar is possible. Equipotential lines are drawn in $50 \mathrm{~V}$ intervals for positive and $500 \mathrm{~V}$ intervals for negative voltages.

$\mathrm{ZrO}_{2}$ or $\mathrm{YSZ}$ (yttria-stabilized $\mathrm{ZrO}_{2}$ ). As there is no gas discharge involved, this type of deposition source can be operated at much lower Ar pressures than magnetrons.

The design by Mayr et al. ${ }^{2}$ comprises a filament at ground potential emitting $\approx 150 \mathrm{~mA}$ and a grid at $+300 \mathrm{~V}$ collecting the electrons. When the vacuum chamber is backfilled with $\mathrm{Ar}, \mathrm{Ar}^{+}$ions created by electron-impact ionization are accelerated onto the sputter target biased at $-2 \mathrm{keV}$. At an Ar pressure of $10^{-4} \mathrm{mbar}$, an ion current of $50-100 \mu \mathrm{A}$ at the target was obtained..$^{2}$ A closer analysis of the design reveals, however, that the electron cloud ionizing the Ar gas [light red in Fig. 1(a)] is mainly at the front side of the grid, where the filament is placed, and at the periphery. $\mathrm{The}^{+} \mathrm{Ar}^{+}$ions generated there bombard the shield at the top side of the sputter source and the surrounding vacuum chamber, which may cause deposition of unwanted material onto the substrate and target as well as desorption of molecules into the residual gas. The maximum kinetic energy of the $\mathrm{Ar}^{+}$ions, when impinging on grounded surfaces, is given by the grid voltage $(300 \mathrm{eV})$. At the other side of the grid, facing the target, the electrons are repelled by the negative high voltage of the target, so ionization is possible only in a rather small volume close to the grid. $\mathrm{Ar}^{+}$ions created at this side of grid near the axis are accelerated to the target for sputtering (the desired effect). It should be noted that the number of ions impinging near the target center could be optimized by placing the target somewhat further away from the grid than shown in Fig. 1(a); the design in Fig. 1(a) is a compromise between optimum sputtering and sufficient heating of the target by thermal radiation from the filament (which is required for sputtering of materials that are insulating at room temperature). ${ }^{6}$

\section{DESCRIPTION OF THE SOURCE}

\section{A. Two-Grid Design}

The problem of only a small fraction of the ionized Ar impinging onto the target can be avoided by using our new design, which has two grids [Fig. 1(b); grids are thin dots in the cross section, only the grid holder rings appear as thick dots]. In the region between the two grids, the electric field directs $\mathrm{Ar}^{+}$ions in the direction towards the target, and the concentric shape of the grids ensures good focusing onto the central area of the target. Nevertheless, some $\mathrm{Ar}^{+}$ions are also created at the other side of the front grid; these ions bombard either the housing of the source or the substrate, see Fig. 1(c). Compared to the original one-grid design, ${ }^{2}$ the efficiency of the two-grid source is significantly better: With grid voltages of +300 and $+200 \mathrm{~V}$ for the front and target-side grid, respectively, a target current of $66 \mu \mathrm{A}$ can be achieved with an emission current of $22+12 \mathrm{~mA}$ (values at the front and rear grid, respectively). This is roughly a quarter of the emission current needed for the same ion current in the onegrid design, at a much lower Ar gas pressure in the UHV system. We used a pressure of $9 \times 10^{-6} \mathrm{mbar} \mathrm{Ar}$ in the vacuum chamber. As the Ar leak valve is connected to the inside of the source housing, the pressure in the source is 27 times higher, about a factor of 2-3 above the pressure used by Mayr et al. $\left(\approx 10^{-4}\right.$ mbar in the UHV chamber, equal to the pressure in the source ${ }^{2}$ ). The ratio of source and chamber pressures can be determined either from the ratio between the pumping speed and conductance of the orifice or from comparing the target current with Ar supplied to the source and that obtained by backfilling the chamber with Ar from a leak valve not connected to the source. These two values agree very well $(<3 \%$ difference).

We can also use lower grid voltages of +150 and $100 \mathrm{~V}$, as shown in Fig. 1(b). This requires a higher filament current (2.4 A through a $0.15 \mathrm{~mm}$ diameter $\mathrm{W}$ wire) and yields a target current of $66 \mu \mathrm{A}$ at $27 \mathrm{~mA}$ emission current (thereof $18 \mathrm{~mA}$ into the front grid and $9 \mathrm{~mA}$ into the back grid). At these conditions, the total power dissipated by electron impact on the grids is only $3.6 \mathrm{~W}$, compared to $45 \mathrm{~W}$ in the original one-grid design. We have also tried higher gas pressures and found that the ion current is proportional to the Ar pressure up to $\approx 2.5 \times 10^{-5} \mathrm{mbar}$ (corresponding to $\approx 7 \times 10^{-4} \mathrm{mbar}$ in the source); at higher pressures the efficiency (ion current at the target per emitted electron and gas pressure) decreases. We could not determine the exact reason for this behavior; possibly it is related to the space charge of the ions. 


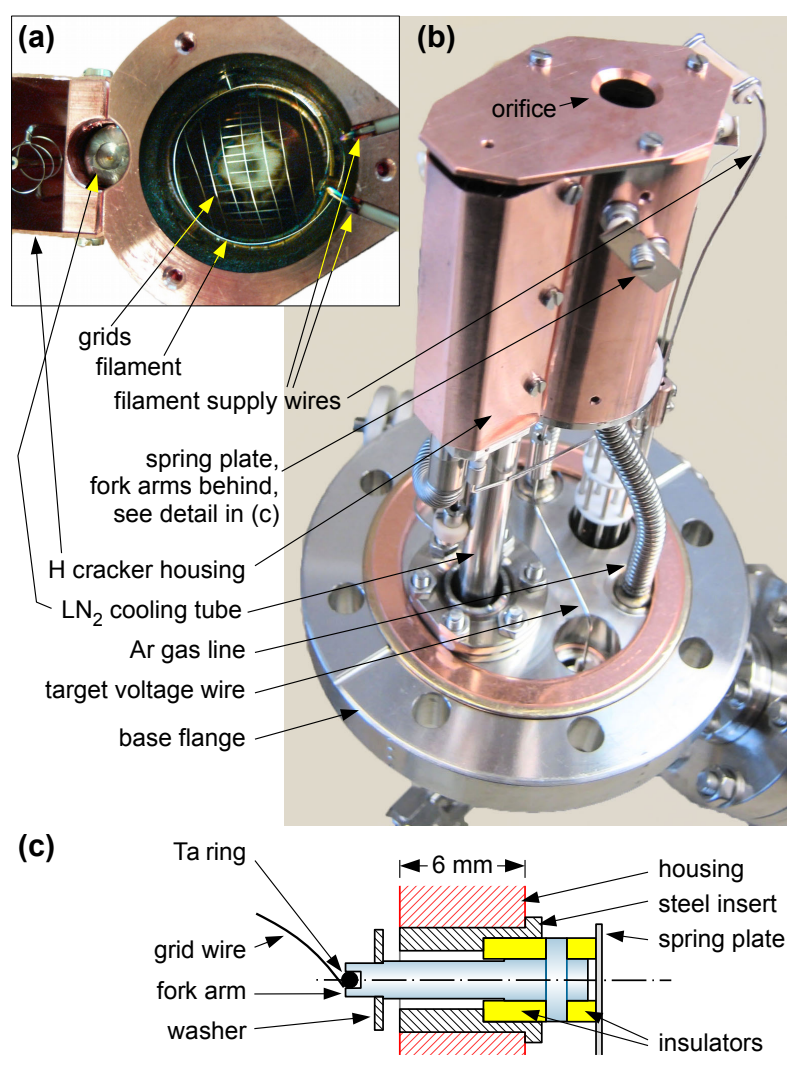

FIG. 2. (a, b) Photos of the sputter source (the hydrogen cracker also seen is unrelated to the function of the sputter source). (a) Front view with the top cover removed; note the bright eroded spot in the middle of the otherwise dark target, where the $\mathrm{Ar}^{+}$ ions are focused to (visible behind the grids). (c) Cross section through the housing at the position of one of the insulated fork arms holding a grid.

\section{B. Design Details}

The source region is enclosed by a housing cooled with liquid nitrogen $\left(\mathrm{LN}_{2}\right)$. With deposition of reactive metals (such as $\mathrm{Zr}$ ) on the inner walls, there is also some pumping of reactive residual-gas molecules (similar to a titanium sublimation pump), which helps to ensure optimum purity of the films deposited. For less stringent purity requirements, the source could also be cooled by water. The housing has the shape of a tube with $24 \mathrm{~mm}$ inner and $36 \mathrm{~mm}$ outer diameter, made of a $\mathrm{CuCrZr}$ alloy (CW106C) with good thermal conductivity (Fig. 2). At the outside, this housing has a groove tightly fitting a stainless steel tube with $8 \mathrm{~mm}$ outer diameter (OD) for cooling [Fig. 2(a)]; a concentric inner tube (3 mm OD) transports the $\mathrm{LN}_{2}$ to the closed end of the $8 \mathrm{~mm}$ tube. The source housing is pressed against the $\mathrm{LN}_{2}$-cooled tube; this tube also serves as support rod to fix the source on the base flange. The end plate of the source at the front side has a $9 \mathrm{~mm}$ hole (orifice for the sputtered material). The back plate contains a high-voltage feedthrough for the target voltage and the Ar gas inlet, connected by hydraulically formed bellows to the weld in the base flange where the tube from the leak valve enters.

The filament is a loop of $0.15 \mathrm{~mm} \mathrm{~W}$ wire, spot welded to the supply wires $(0.8 \mathrm{~mm} \mathrm{Ta})$; the negative terminal of the filament supply is at ground potential. The grids have the shape of spherical caps, with the center of curvature roughly in the center of the target, to focus the $\mathrm{Ar}^{+}$ions to the target center [see the bright erosion spot in the center of the target in Fig. 2(a)]. This yields a uniform deposition rate on the substrate, without vignetting. The grids are made from $0.175 \mathrm{~mm} \mathrm{~W}$ wire spot-welded onto rings ( $\approx 20 \mathrm{~mm}$ outer diameter) made from $0.8 \mathrm{~mm}$ Ta wire. A previous attempt to make the rings from stainless steel was unsuccessful; at high power $(+300 \mathrm{~V}$ at the front grid), the steel ring got hot enough to soften and deform, finally touching the housing of the source. The rings of the grids are held by fork-like arms (three per grid); these protrude through the source housing and are held by ceramic insulators [Fig. 2(c)]. Washers protect the insulators from sputtered material scattered by the Ar gas. For mounting the grids inside the housing, the grids have to be temporarily held in position from the open end of the source housing, then the forks are inserted radially from the outside. Spring plates (one of them marked in Fig. 2) prevent the forks from sliding back. For Zr deposition, the target is a disk of $\mathrm{Zr}$ metal (diameter $18 \mathrm{~mm}$, thickness $1 \mathrm{~mm}$ ) with $99.9 \%$ purity (HMW Hauner, Germany). The target was spot welded to a support rod at its back side; the rod is held by the high-voltage feedthrough in the back plate of the source housing.

The source is placed on a DN63CF base flange (4.5" outer diameter). The base flange has DN16CF (1.25" outer diameter) ports for the electrical feedthroughs, the $\mathrm{LN}_{2}$ tube and the Ar gas. As the source is rather compact, there is enough space for further components on the same base flange. We have added a hydrogen cracker similar to Refs. 7 and 8; the housing of the $\mathrm{H}$ cracker is cooled together with the sputter source. In addition, for future extensions, there is some space for mounting further sources, e.g. tiny crucibles for evaporation, at the sides of the sputter source (to make space, two sides of the source housing are milled flat, as visible in Fig. 2(a).

\section{EVALUATION OF THE SOURCE}

\section{A. Deposition Rate}

We can calculate the deposition rate $F$ from the source by assuming a cosine (Lambertian) angular distribution of the sputtered atoms. Assuming an incident ion current $I_{\mathrm{sp}}$, a sputter yield $Y$, and $r$ being the distance between the target and the substrate, we obtain

$$
F(r)=\frac{I_{\mathrm{sp}} Y}{r^{2} \pi|e|}=\frac{I_{\mathrm{t}} Y}{r^{2} \pi|e|(1+\gamma)}
$$

for emission perpendicular to the target, which is the direction to the substrate (Fig. 1). The charge of the ions is $|e|$, and the factor $(1+\gamma)$ in the denominator of the last term accounts for the fact that the measured target current $I_{\mathrm{t}}$ is higher than that of the incident ions $\left(I_{\mathrm{sp}}\right)$ due to ion-induced electron emission, with $\gamma$ being the electron yield upon ion impact. Equation (1) neglects the effects of resonant neutralization of fast $\mathrm{Ar}^{+}$ions by collisions 
with neutral Ar. This is justified when considering the short path of the $\mathrm{Ar}^{+}$ions $(1-2 \mathrm{~cm})$. With a target current of $66 \mu \mathrm{A}, r=50 \mathrm{~mm}, Y=1.15$ (for $2 \mathrm{keV} \mathrm{Ar}^{+} \rightarrow \mathrm{Zr}$, Ref. 9 ), and $\gamma$ in the range of 0.1-0.2 (consistent with the measured electron current at the substrate, see below), Eq. (1) yields a deposited flux $F$ between 5 and $5.5 \times 10^{12}$ atoms per $\mathrm{cm}^{2}$ and second.

Since the deposition rate determined with a quartz crystal microbalance (QCM) is not very accurate in this case (see below), we have determined the deposition rate by submonolayer growth of $\mathrm{Zr}$ on a well-defined singlecrystal substrate, $\mathrm{Rh}(111)$, and determined the area covered by $\mathrm{Zr}$ islands by scanning tunneling microscopy (STM). To determine the deposition rate from the Zrcovered area, we assume that the density of $\mathrm{Zr}$ atoms in the islands is equal to that in the basal plane of $\mathrm{Zr}$, $1.11 \times 10^{15} \mathrm{~cm}^{-2}$. The analysis of the STM images yields a deposition rate of $4.5 \times 10^{12} \mathrm{~cm}^{-2} \mathrm{~s}^{-1}$. Considering the uncertainty of the sputter yield and the angular distribution, the agreement must be considered excellent. As the quantities in Eq. 1 are not expected to change with time, this also indicates that the sputter source should have excellent stability of the deposition rate, which perfectly agrees with our experience (provided that the sputter target is sufficiently clean, which is usually the case after a few minutes of operation). The deposition rate remains also unchanged when the source is operated at an additional oxygen partial pressure of $10^{-6}$ mbar for growing $\mathrm{ZrO}_{2}$ films (we monitored the reproducibility of the deposition rate by checking for completion of the $5^{\text {th }} \mathrm{ZrO}_{2}$ layer with STM; this should be accurate within a few percent).

\section{B. Purity of the Films}

Figure 3(a-c) shows x-ray photoelectron spectra (XPS) of a clean $\mathrm{Rh}(111)$ surface and immediately after deposition of $2.9 \mathrm{ML} \mathrm{Zr}$ with the UHV sputter source. ${ }^{10} \mathrm{We}$ define $1 \mathrm{ML}$ as the areal density of $\mathrm{Zr}$ in the basal plane $\left(1.11 \times 10^{15} \mathrm{~cm}^{-2}\right)$. The $\mathrm{Rh}(111)$ substrate was at room temperature during deposition and the measurements. The spectra were acquired with a non-monochromatized Mg K $\alpha$ source and a Specs Phoibos 100 energy analyzer in an analysis chamber coupled to the chamber with the sputter source (the base pressure in both chambers is below $10^{-10} \mathrm{mbar}$ ). The only impurity detectable is a small amount of oxygen, probably from oxygen implanted into the $\mathrm{Zr}$ target or dissolved there during many previous experiments at $10^{-6}$ mbar $\mathrm{O}_{2}$ for growing $\mathrm{ZrO}_{2}$ films. The $\mathrm{C} 1 \mathrm{~s}$ region does not show any indication of carboncontaining impurities; the small peaks found in this range after $\mathrm{Zr}$ deposition are due to excitation of stronger lines by satellite lines of the x-ray source, as well as Ru, which is an impurity in our Rh substrate [Fig. 3(b)]. Zooming in onto the $\mathrm{Zr} 3 \mathrm{~d}$ lines shows only metallic $\mathrm{Zr}$ peaks [Fig. $3(\mathrm{c})]$. The $\mathrm{Zr} 3 \mathrm{~d}_{5 / 2}$ binding energy of $179.4 \mathrm{eV}$ is slightly higher than usually reported for pure $\mathrm{Zr}$ bulk (178.7$178.9 \mathrm{eV})$. We attribute this peak shift partly to the surface core level shift, which is about half an $\mathrm{eV}$ to higher $\mathrm{BE}$ for close-packed surfaces of the early $4 \mathrm{~d}$ transition
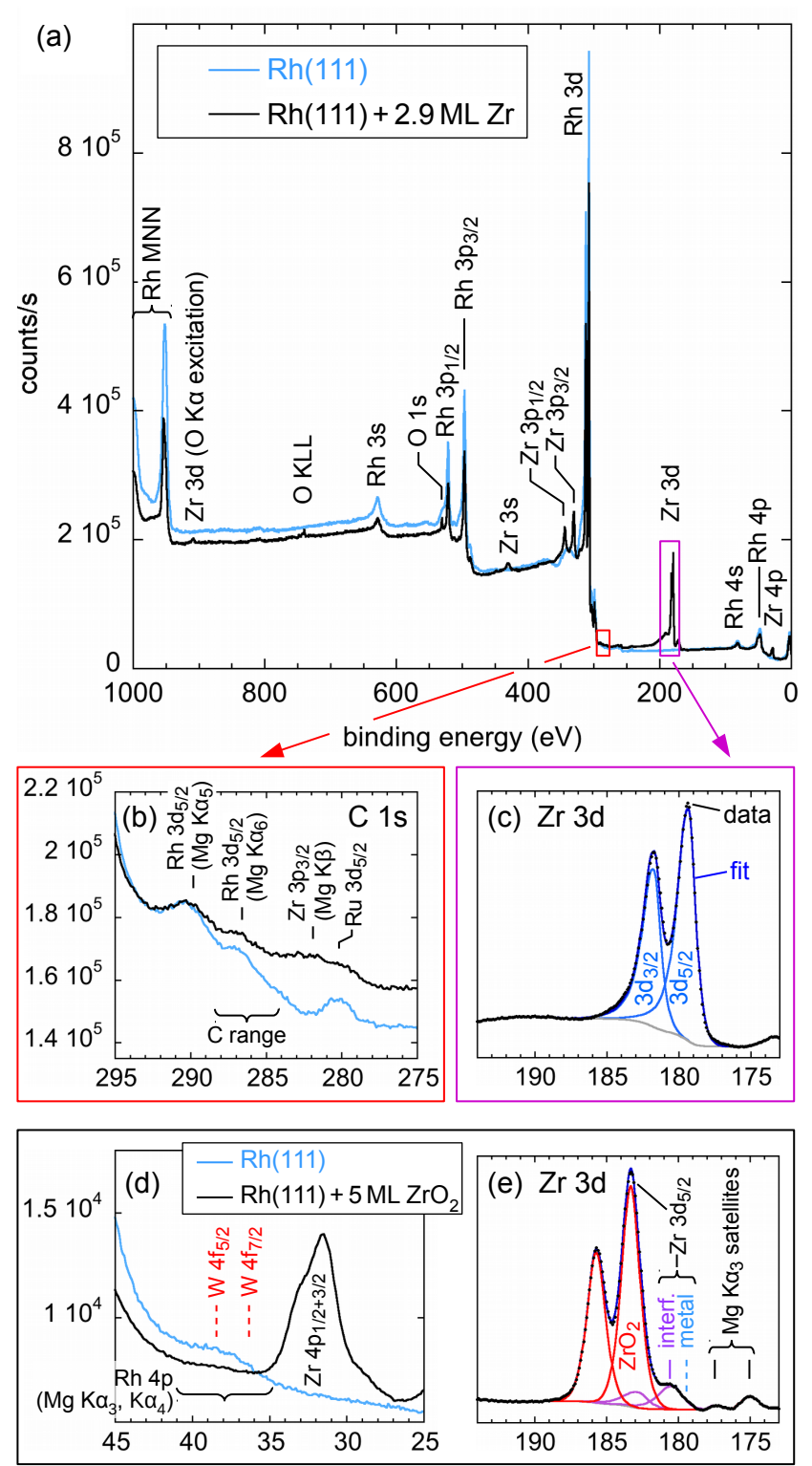

FIG. 3. (a-c) XPS of a clean Rh(111) surface and after deposition of $2.9 \mathrm{ML}(0.74 \mathrm{~nm}) \mathrm{Zr}$. The $\mathrm{C} 1 \mathrm{~s}$ region (b) was measured with high sensitivity; the range of typical carbon impurities (adventitious carbon and species with $\mathrm{C}-\mathrm{O}$ bonds) is indicated. The detailed spectrum of the $\mathrm{Zr} 3 \mathrm{~d}$ range (c) shows only metallic $\mathrm{Zr}$ $\left(\mathrm{Zr} 3 \mathrm{~d}_{5 / 2}\right.$ at $\left.179.4 \mathrm{eV}\right)$ as indicated by the fit; a slight shoulder to the left, if any, has very low intensity. Spectra obtained after $\mathrm{Zr}$ deposition in $10^{-6} \mathrm{mbar}_{2}[1.0 \mathrm{~nm} \mathrm{Zr}$ corresponding to $5 \mathrm{ML}$ $\left.\mathrm{ZrO}_{2}(111)\right]$ show (d) the absence of (oxidized) tungsten and (e) only oxidic $\mathrm{Zr}$ peaks. Analyzer pass energy (a) $20 \mathrm{eV}$ (b) $50 \mathrm{eV}$ (for increased count rate); in all other spectra $16 \mathrm{eV}$.

metals. ${ }^{11,12}$ A further contribution will come from interaction of $\mathrm{Zr}$ with $\mathrm{Rh}$ at the interface, as $\mathrm{Zr} 3 \mathrm{~d}_{5 / 2}$ energies of $\approx 179.6 \mathrm{eV}$ are found for $\mathrm{Zr}$ alloys with other late transition metals $\left(\mathrm{Pt}_{3} \mathrm{Zr}\right.$ and $\mathrm{Pd}_{3} \mathrm{Zr}$, Refs. 13 and 14). To some degree, the shift may be also related to interaction with oxygen impurities. In addition, curve fitting cannot exclude an extremely weak shoulder to the left ( $\mathrm{Zr}$ $3 \mathrm{~d}_{3 / 2}$ at $184-184.5 \mathrm{eV}$, corresponding to $3 \mathrm{~d}_{5 / 2}$ at $\approx 182 \mathrm{eV}$ ), possibly also related to oxygen: Ultrathin and bulk-like $\mathrm{ZrO}_{2}$ would have the $\mathrm{Zr} 3 \mathrm{~d}_{5 / 2}$ peaks at 180.7 and around 
$182.8 \mathrm{eV}$, respectively. ${ }^{13}$

Figure 3(d, e) shows spectra obtained after Zr deposition on $\mathrm{Rh}(111)$ at an additional oxygen partial pressure of $10^{-6}$ mbar at room temperature. At these conditions, a $\mathrm{ZrO}_{2}$ film grows, but without post-annealing the oxide is poorly ordered. The main peak at $183.3 \mathrm{eV}$ is from $\mathrm{ZrO}_{2}$ (due to the large band gap of $\approx 5 \mathrm{eV}$, the band alignment and, thus, the binding energies can shift considerably). There is also a minor peak at $180.4 \mathrm{eV}$, which decreases with increasing film thickness, thus it must come from $\mathrm{Zr}$ at the interface. As this peak does not disappear upon annealing under oxidizing conditions, we attribute it to $\mathrm{ZrO}_{2}$, not to a lower oxidation state of $\mathrm{Zr}$, in agreement with DFT calculations that predict a lower $\mathrm{Zr} 3 \mathrm{~d}$ binding energy at the $\mathrm{ZrO}_{2}$-metal interface. ${ }^{13}$ The spectrum shows no metallic Zr. Mayr et al. have reported that their source, when operated with $\mathrm{O}_{2}$ in the background gas, can lead to tungsten and tantalum impurities in the films, presumably from formation of volatile oxides on the filament and grid materials (W and Ta, respectively; due to the high power these grids get very hot). ${ }^{2}$ We also checked for tungsten impurities coming from the filament or grid wires (in our source, both are W). Fig. 3(d) shows no indication of any $\mathrm{W}$ signal at the positions where it was observed by Mayr et al. (dashed lines in the figure). W would show a sharp doublet there, superimposed on the broad $\mathrm{Rh} 4 \mathrm{p} \mathrm{Mg} \mathrm{K} \alpha_{3}+\mathrm{K} \alpha_{4}$ satellite, thus we can exclude such a problem for our source.

\section{C. $\mathrm{Ar}^{+}$Ion and Electron Emission}

Apart from sputtered target material, two types of particles are emitted from the source [Fig. 1(c)]: $\mathrm{Ar}^{+}$ions and electrons. As mentioned above, Ar gas ionized in the region above the front grid will not be accelerated towards the target but rather to the end plate with the orifice or out of the source, towards the substrate. These ions can sputter material from the inside of the source housing onto the target. Fortunately, after short operation of the source, all the inside of the housing is covered with target material, so there is no contamination of the target by wall material. Some $\mathrm{Ar}^{+}$ions (with energies in $\mathrm{eV}$ up to the voltage of the front grid) also reach the substrate; these $\mathrm{Ar}^{+}$ions will lead to mild sputtering of the target. Indeed, in our experiments with sputter deposition of $\mathrm{Zr}$ onto well-prepared $\mathrm{Rh}(111)$ single crystals, we have observed the formation of vacancy islands in the Rh surface. The scanning tunneling microscopy (STM) images in Figure 4(a) show the vacancy islands as small black patches. These vacancy islands can be explained only by sputtering of the sample, which happens in addition to the deposition of sputtered target material. In some cases, this effect can be desired (ion-beam assisted deposition, IBAD) ${ }^{1,15}$. Impingement of particles with energies above $\approx 150 \mathrm{eV}$ leads to an increased density of nuclei, which can promote layer-by-layer growth in some cases. ${ }^{16} \mathrm{~A}$ disadvantage of mild sputtering is reduced accuracy of the deposition rate due to mass removal. When growing oxides or compound materials, preferential sputtering will also alter the composition of the film. For the experiment

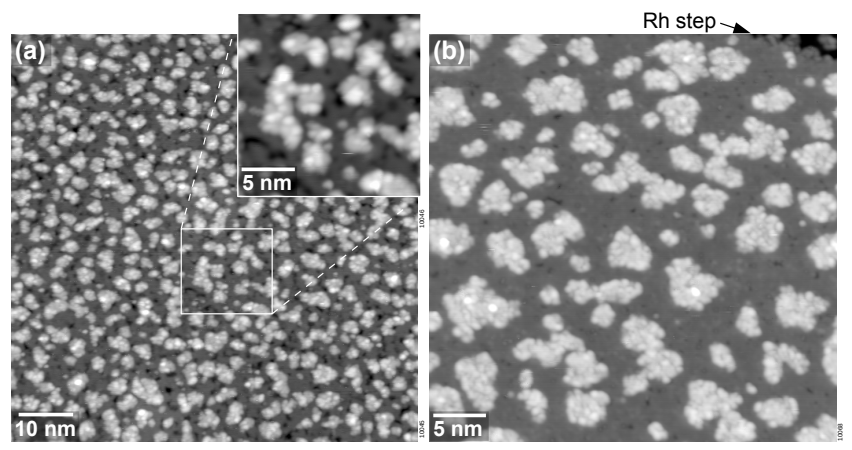

FIG. 4. STM images of a Rh(111) surface after deposition of 0.35 ML of Zr with front/rear grid voltages of (a) $300 \mathrm{~V} / 200 \mathrm{~V}$ and (b) $150 \mathrm{~V} / 100 \mathrm{~V}$. Vacancy islands appear as small black patches in the images. The sputter damage can be reduced drastically by reducing the grid voltages.

shown in Fig. 4(a) the grid voltages were 300 and $200 \mathrm{~V}$ for the front and rear grid, respectively $\left(\mathrm{Ar}^{+}\right.$energies up to $300 \mathrm{eV})$. We usually choose grid voltages of 150 and $100 \mathrm{~V}$. In this case, the sputter yield $Y$ at the maximum ion energy $(150 \mathrm{eV})$ is sufficiently low to avoid these problems (e.g., $Y \approx 0.34$ for $150 \mathrm{eV} \mathrm{Ar}{ }^{+} \rightarrow \mathrm{Rh}$, Ref. 9). This is also seen in Fig. 4(b), where only very few and small vacancy islands are found.

The sputter deposition source also emits electrons, which are liberated by ion impact at the target [Fig. 1(c)]. These electrons are accelerated to $2 \mathrm{keV}$ by the target voltage. Quantification of the electron current on the substrate is not easy, because only the sum of the electron and $\mathrm{Ar}^{+}$ion current can be measured; the emission of secondary electrons upon $2 \mathrm{keV}$-electron impact at the substrate further complicates the problem. The substrate current is given by

$$
I_{\text {substr }}=I_{\text {ion }}+I_{\mathrm{el}}(1-\delta)
$$

where $\delta$ is the secondary electron yield for electron impact at the substrate (due to the low ion energies, ion-induced electron emission from the substrate can be neglected). We have studied this effect with a Pt(111) single crystal serving as substrate. In the initial phase of the deposition, while the substrate was essentially uncovered, we have measured a substrate current of $I_{\mathrm{Pt}}=2.2 \mu \mathrm{A}$; with increasing $\mathrm{Zr}$ coverage it was found to decrease to $I_{\mathrm{Zr}}=-1.2 \mu \mathrm{A}$. With these two values, and $\delta=1.22(0.51)$ for $\mathrm{Pt}(\mathrm{Zr})$ at $2 \mathrm{keV},{ }^{17}$ we obtain an electron current of

$$
I_{\mathrm{el}}=-\frac{I_{\mathrm{Pt}}-I_{\mathrm{Zr}}}{\delta_{\mathrm{Pt}}-\delta_{\mathrm{Zr}}} \approx-4.8 \mu \mathrm{A}
$$

and an ion current of $\approx 1.1 \mu \mathrm{A}$. Eq. (3) contains the difference between two secondary electron yield values $\delta$, which are not known with high accuracy; the exact value of $\delta$ may also depend on the crystallographic properties of the material and electron scattering in the layers below (Pt single crystal and $\mathrm{Zr}$ thin film in our experiment vs. $\delta$ values of polycrystalline material in the literature). Thus, some uncertainty of the electron current is to be expected. This uncertainty has a significant impact on the ion current, which is calculated as the difference of two larger 
quantities according to Eq. (2). We have also tried to determine the secondary electron yields with a $2 \mathrm{keV}$ electron source from the difference between the sample current with and without a positive $30 \mathrm{~V}$ sample bias, yielding $\delta_{\mathrm{Pt}}=1.82$ and $\delta_{\mathrm{Zr}}=0.68$. These values would result in $I_{\mathrm{el}}=-3.0 \mu \mathrm{A}$, but a negative ion current $(-0.2 \mu \mathrm{A})$. Thus, the ion current cannot be determined with reasonable accuracy by this method. Based on the sputter damage observed by STM, we estimate that the ion current is actually in between these two values; i.e. a few tenths of a $\mu \mathrm{A}$.

Due to the high kinetic energy of the electrons, they dissipate a power of $\approx 10 \mathrm{~mW}$ at the substrate. This is not an issue for most substrates, but it affects the reading of a quartz crystal microbalance used to determine the deposition rate. In our experience, with standard ATcut $6 \mathrm{MHz}$ quartz crystals used for thin-film deposition monitors $^{18}$ and a water-cooled crystal holder, the time for equilibration (until a stable deposition rate is displayed) is about 10-20 min for thermal evaporation, but about an hour with the sputter deposition source (SCcut quartz crystals, which are also used for sputter yield measurements, ${ }^{19}$ are less sensitive to thermal stress and would perform better). In our experience, even after equilibration the QCM readings scatter by more than $10 \%$. We rather rely on the deposition rate determined once (months ago) from the coverage determined by STM. To ensure best reproducibility, we adjust the gas pressure to get the same target current each time. In other words, the reproducibility of the deposition rate of the sputter source (estimated to be better than a few percent over several months, including several bakeout cycles of the UHV system) is better than that of the $\mathrm{QCM}$ readings.

In case that electron or ion emission causes a substantial problem, it would be possible to add an extra electrode at the orifice to repel or deflect these charged species. If the position of the front end of the source is constrained by having to avoid collisions with the sample holder, this would slightly increase the target-to-substrate distance and thereby reduce the deposition rate. If the substrate can be biased, this would provide another possibility to repel either electrons or ions (at the cost of increased kinetic energy of the other species).

\section{CONCLUSIONS}

We have presented a sputter deposition source optimized for growth of clean films in ultrahigh vacuum. Compared to the design by Mayr et al. ${ }^{2}$, the source operates at more benign values of filament and emission current and lower Ar gas pressures in the UHV chamber for comparable target current and deposition rate. If desired, higher deposition rates could be achieved by increasing the gas pressure or emission current. The source also features excellent long-time reproducibility and the films grown show very high purity. During deposition, the substrate is subject to a flux of both electrons and low-energy $\mathrm{Ar}^{+}$ions. The energy of the latter can be controlled by the grid voltages, so the source provides the possibility of either ion-beam-assisted deposition or negligible sputtering of the substrate and films by $\mathrm{Ar}^{+}$ions. Apart from materials that are difficult to evaporate in UHV (such as $\mathrm{Zr}$ ), due to the high purity of the films grown we consider our source a good choice also for many other materials, especially when considering the advantages of sputter deposition mentioned in the introduction.

\section{ACKNOWLEDGMENTS}

The authors would like to thank Peter Varga for helpful discussions, Herbert Schmidt and Rainer Gärtner for the machine shop work and fruitful discussions about construction details, and Martin Leichtfried for designing some of the parts and assembling the source. This work was supported by the Austrian Science Fund (FWF) under project number F4505 (Functional Oxide Surfaces and Interfaces - FOXSI).

\footnotetext{
${ }^{1}$ T. Michely, M. Kalff, and G. Comsa, Mat. Res. Soc. Symp. Proc. 528, 179 (1998).

${ }^{2}$ L. Mayr, N. Köpfle, A. Auer, B. Klötzer, and S. Penner, Rev. Sci. Instrum. 84, 094103 (2013).

${ }^{3}$ T. Götsch, W. Wallisch, M. Stöger-Pollach, B. Klötzer, and S. Penner, AIP Advances 6, 025119 (2016).

${ }^{4}$ C. Schwebel, F. Meyer, G. Gautherin, and C. Pellet, Journal of Vacuum Science \& Technology B: Microelectronics Processing and Phenomena 4, 1153 (1986).

${ }^{5}$ N. Lee, G. Xue, and J. E. Greene, Journal of Applied Physics 80, 769 (1996).

${ }^{6}$ B. Klötzer and S. Penner, private communication.

${ }^{7}$ U. Bischler and E. Bertel, J. Vac. Sci. Technol. A 11, 458 (1993).

${ }^{8}$ C. Eibl, G. Lackner, and A. Winkler, J. Vac. Sci. Technol. A 16, 2979
} (1998).

${ }^{9}$ N. Matsunami, Y. Yamamura, Y. Itikawa, N. Itoh, Y. Kazumata, S. Miyagawa, K. Morita, R. Shimizu, and H. Tawara, "Energy dependence of the yields of ion-induced sputtering of monatomic solids," Tech. Rep. IPPJ-AM-32 (Institute of Plasma Physics, Nagoya University, Japan, 1983).

${ }^{10}$ We have used $99.999 \%$ Ar (CANgas, www.messergroup.com).

${ }^{11}$ R. G. Jordan, A. M. Begley, S. D. Barrett, P. J. Durham, and W. M. Temmerman, Sol. State Commun. 76, 579 (1990).

${ }^{12}$ M. Methfessel, D. Hennig, and M. Scheffler, Surf. Sci. 287, 785 (1993).

${ }^{13}$ H. Li, J.-I. J. Choi, W. Mayr-Schmölzer, C. Weilach, C. Rameshan, F. Mittendorfer, J. Redinger, M. Schmid, and G. Rupprechter, J. Phys. Chem. C 119, 2462 (2015).

${ }^{14}$ J. I. J. Choi, W. Mayr-Schmölzer, F. Mittendorfer, J. Redinger, U. Diebold, and M. Schmid, J. Phys.: Condens. Matter 26, 225003 (2014).

${ }^{15}$ J. M. E. Harper, J. J. Cuomo, R. J. Gambino, and H. R. Kaufman, Nucl. Instrum. Meth. B 7-8, 886 (1985).

${ }^{16}$ M. Schmid, C. Lenauer, A. Buchsbaum, F. Wimmer, G. Rauchbauer, P. Scheiber, G. Betz, and P. Varga, Phys. Rev. Lett. 103, 076101 (2009).

${ }^{17}$ Y. Lin and D. C. Joy, Surf. Interface Anal. 37, 895 (2005).

${ }^{18}$ E. Benes, M. Gröschl, W. Burger, and M. Schmid, Sensor. Actuator. A 48, 1 (1995).

${ }^{19}$ G. Hayderer, M. Schmid, P. Varga, H. Winter, and F. Aumayr, Rev. Sci. Instrum. 70, 3696 (1999). 